\title{
Uterine AV malformation and treatment related Fertility
}

\section{outcome}

\section{Lubna $\mathrm{K}^{1,2 *}$, Megren Al B ${ }^{1,2}$, Eman Al Z2 ${ }^{2}$, Mariam J33 ${ }^{3}$ Hafiza $\mathrm{T}^{1,2}$ and} Shoeb $\mathbf{Q}^{1,2}$

${ }^{1}$ King Saud bin Abdul-Aziz University for Health Sciences, King Abdul-Aziz Medical City and King Abdullah International Medical Research Centre, Saudi Arabia

${ }^{2}$ King Abdul-Aziz Medical City and King Abdullah International Medical Research Centre, Saudi Arabia

${ }^{3} \mathrm{Al}$ Faisal university, Saudi Arabia

*Corresponding author: Lubna Khan, King Saud bin Abdul-Aziz University for Health Sciences, King Abdul-Aziz Medical City and King Abdullah International Medical Research Centre, Saudi Arabia, Email: lubnamurshid@yahoo.com

\section{Abstract}

The incidence and prevalence of uterine arteriovenous malformations (AVMs) is difficult to determine because bleeding caused by uterine AVMs does respond to medical management and several of these may go undiagnosed. Fewer than 100 cases have been reported in the literature, but the increased use of imaging modalities, particularly ultrasound in acute gynecology is likely to lead to identification of these lesions more frequently. Uterine AVMs are mainly acquired lesions, with pregnancy playing an important role in the pathogenesis. The most frequent clinical manifestation is abnormal uterine bleeding, which can often be episodic, heavy and can result in significant anemia or even shock. Hysterectomy remains the most definitive treatment; however, modern management of uterine AVMs includes medical management (hormonal therapy), minimally invasive surgery, uterine artery embolization and even Hysterectomy. As women who has AVM are largely from reproductive age group, effect of different treatment modalities especially uterine artery embolisation on fertility is still a subject of debate.

Keywords: Conservative Treatment Options; Diagnosis; Lesions; Uterine Arteriovenous Malformations; Uterine Bleeding; Uterine Artery Embolization

\section{Introduction}

Uterine arteriovenous malformation (AVM) is a vascular hamartoma of the myometrium characterized by the presence of shunts between the myometrial arteries and veins. Most AVMs are acquired after damage to uterine tissue. A miscarriage, dilation and curettage
(D\&C), cesarean section, vaginal delivery and carcinoma of the cervix or endometrium as well as uterine infection, trophoblastic disease, endometriosis and exposure to diethylstilbestrol are among the reported causes of AVM [1-3]. 


\section{Medical Journal of Clinical Trials \& Case Studies}

Congenital AVM develop due to failure of embryological differentiation of primitive vascular structures, resulting in the creation of abnormal vascular connection [4]. Histologic examination of these malformations reveals many thin-walled capillaries intertwining with the vessels in the myometrium. Congenital AVM tend to extend beyond the margins of the uterus into the pelvis, with frequent feeders from pelvic vessels other than the uterine artery. These lesions have been predominantly reported in women of reproductive age but very rarely in nulliparous [5].

The most frequent presenting symptom of AVM is profuse menorrhagia or metrorrhagia that does not respond to medical treatment, eventually leading to anemia. The other symptoms are lower abdominal pain and dyspareunia. Untreated Uterine AVM has been linked to recurrent pregnancy loss, because of the hyper vascularization that may alter the implantation site of the embryo, but there is no issue of infertility or intrauterine growth restriction [6].

There is no clear consensus on the best treatment for AVM [7]. The current medical and surgical options are based on clinician's experience and published case reports. The treatment depends on the extent of bleeding. In mild to moderate cases with Hemodynamically stable patients, the first clinical approach would be conservative for a maximum period of 3-6 months [8]. Conservative management consists of medical therapy with methylergonovine maleate, danazol, and Gn-RH agonists and it is associated with a high rate of failure. Persistent bleeding and failed conservative management indicate embolisation of the uterine or iliac artery which effectively treats the lesion [9]. Embolisation is effective in $57 \%$ of cases and a second embolisation may be necessary in up to $32 \%$ of patients for persistent bleeding $[10,11]$. Major risks for the patient after embolization of the uterine artery are post embolisation syndrome (massive necrosis and infarction of the uterus, uterine artery rupture, and pelvic pain), transient or permanent amenorrhea, and radiation exposure $[12,13]$. There are other less frequently used surgical methods, such as the coagulation of AVM under hysteroscopic guidance, laparoscopic bipolar coagulation of uterine vessels and surgical removal of the AVM [2,14-16].

\section{Case Report}

23 years old primigravidae 38 weeks of gestation, presented to labor and delivery suite in active labor. She had uneventful antenatal follow up and considered low risk. Patient delivered uneventfully with right mediolateral episiotomy and a lateral vaginal wall tear. She had active management of the $3^{\text {rd }}$ stage and completely delivered the placenta. Post- delivery patient started to bleed heavily. On examination uterus was well contracted and Bedside scan revealed empty uterine cavity.

After failed medical management Patient was taken to Operation theatre for examination under anesthesia and repair of deep vaginal tear. Exploration and Repair of the vaginal tear and episiotomy done but patient continue to have on and off bleeding despite a well contracted uterus. Vaginal pack was inserted in view of some oozing from the suture line, which was supposed to be removed after 12 hours. Patient was then transferred to the High dependency unit. After few hours she gets hemodynamic ally unstable because of continuous bleeding.

Obstetric tem was called up and Case was discussed with interventional radiologist and planned for internal iliac artery embolization. A pelvic angiogram was performed which noticed large abnormal vascular flow from the right side of the uterine cavity supplied mainly through branches of the internal iliac artery. Uterine Atriovenous malformation was suspected and internal iliac artery embolisation was performed. After the embolisation bleeding controlled and rest of her stay in the hospital went smooth with good recovery. She was discharged from the hospital after four days but didn't show up afterwards for her follow-up appointments.

After six months she presented to accident and emergency department with history of 12 weeks amenorrhea, abdominal pain and heavy vaginal bleeding. Bhcg was $830 \mathrm{iu} / \mathrm{l}$ on ultrasound there was no intrauterine gestational sac seen and the endometrium was thick, Endo- cavity measured $5 \mathrm{~cm}$ with multiple cystic areas and high vascularity. Pelvic MRA done showed thickened posterior myometrium with a multiple serpentine flow void enhancing structures identified within the myometrium and reaching till the endometrium, picture was highly suggestive of underlying atriovenous malformation. Uterine artery embolisation was performed. Patient had repeated MRI scan afterwards at regular intervals that showed complete resolution of the AVM. She got pregnant again and had a tubal ectopic which was treated medically with Methotrexate. 


\section{Medical Journal of Clinical Trials \& Case Studies}
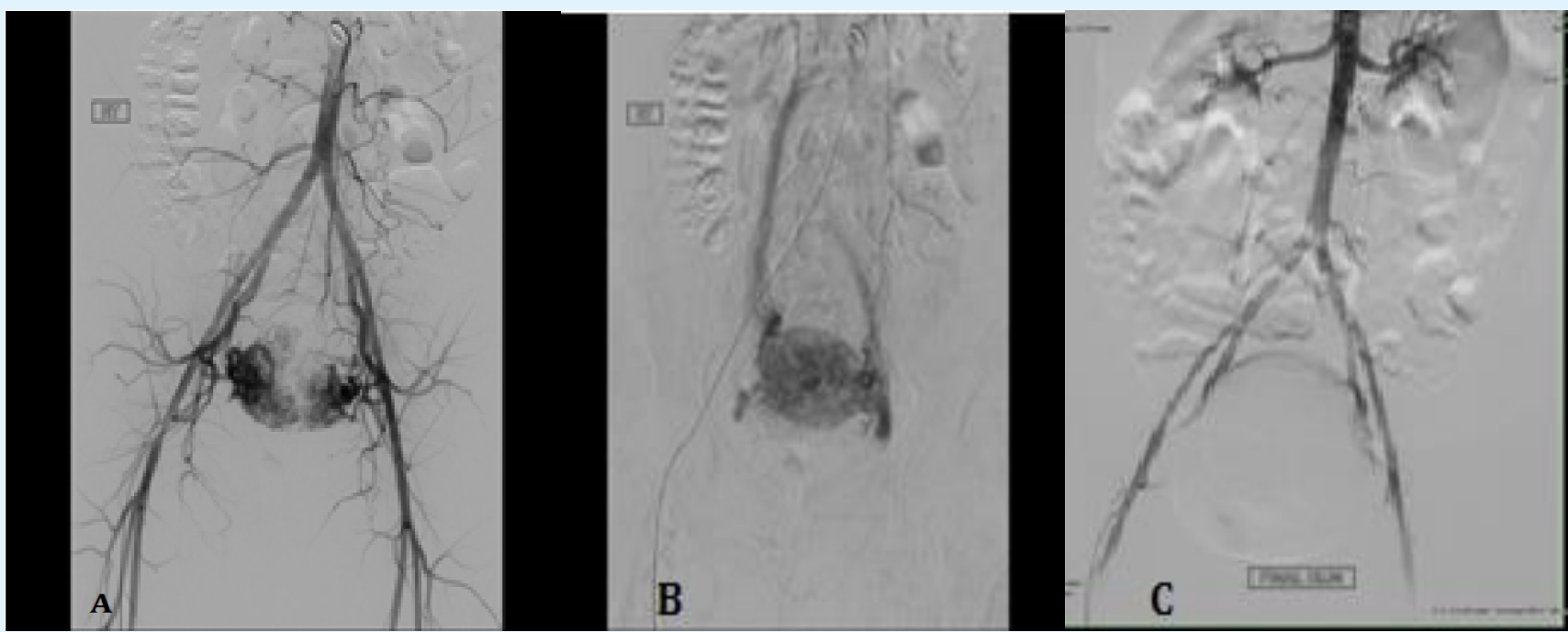

Figure 1: A: Abdominal and pelvic angiogram showing hypervascularity of uterine arteries.

B: Abdominal and pelvic angiogram showed early venous drainage in keeping with uterine arteriovenous malformation.

C: Final angiogram of the abdomen and pelvis post embolization using gelatin sponge slurry showed completely embolized uterine arteriovenous malformation.

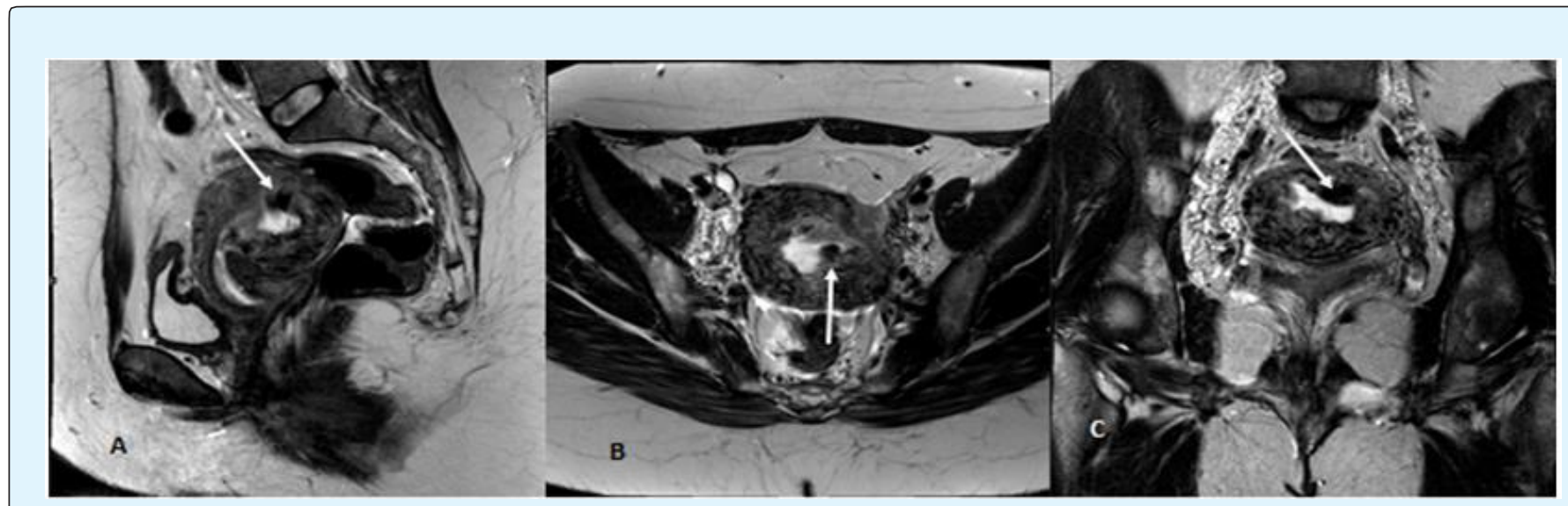

Figure 2: Sagittal (A); Axial (B) and Coronal (C): T2-wieatghed images of the pelvis at the level of the uterus show abnormal hypo intense focus/flow void along the anterior upper endometrial cavity that extend into the myometrial (white arrow in A, B and C). Note the retroverted retroflexed uterus. 


\section{Medical Journal of Clinical Trials \& Case Studies}

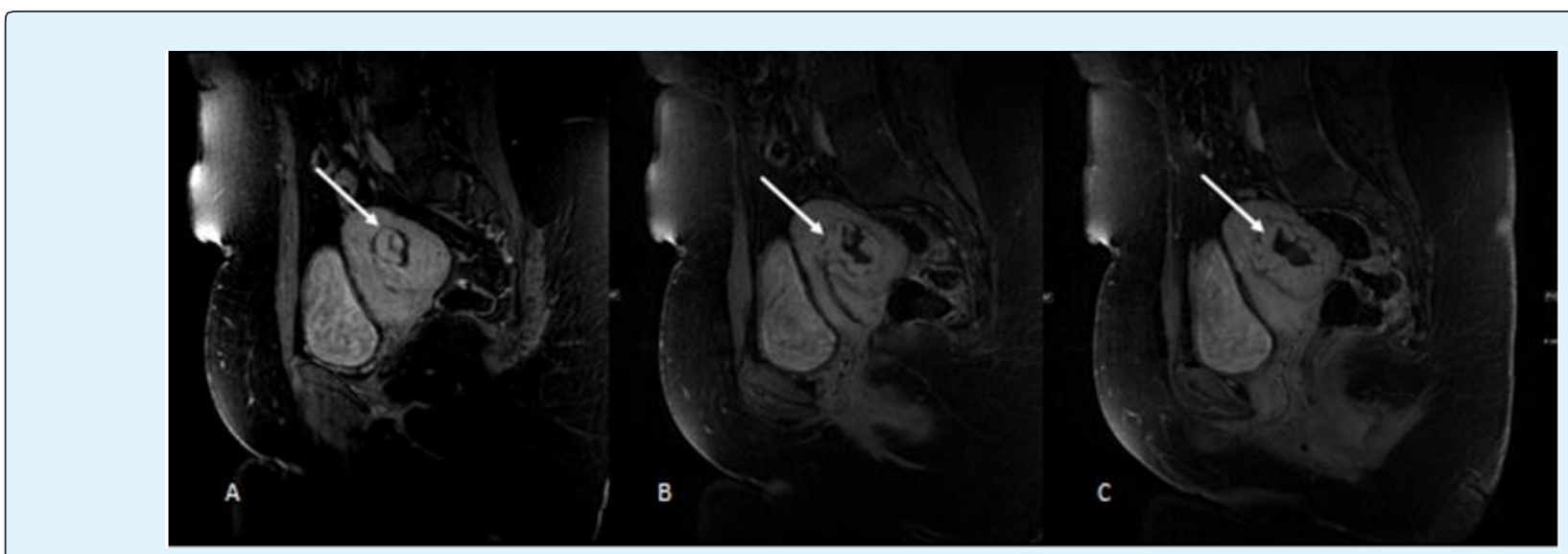

Figure 3: Multiple sagittal contrast-enhanced pelvic MR show multiple large serpentine abnormal vesselsa along the endometrial cavity that extend into the myometrium (white arrow in A, B and C).

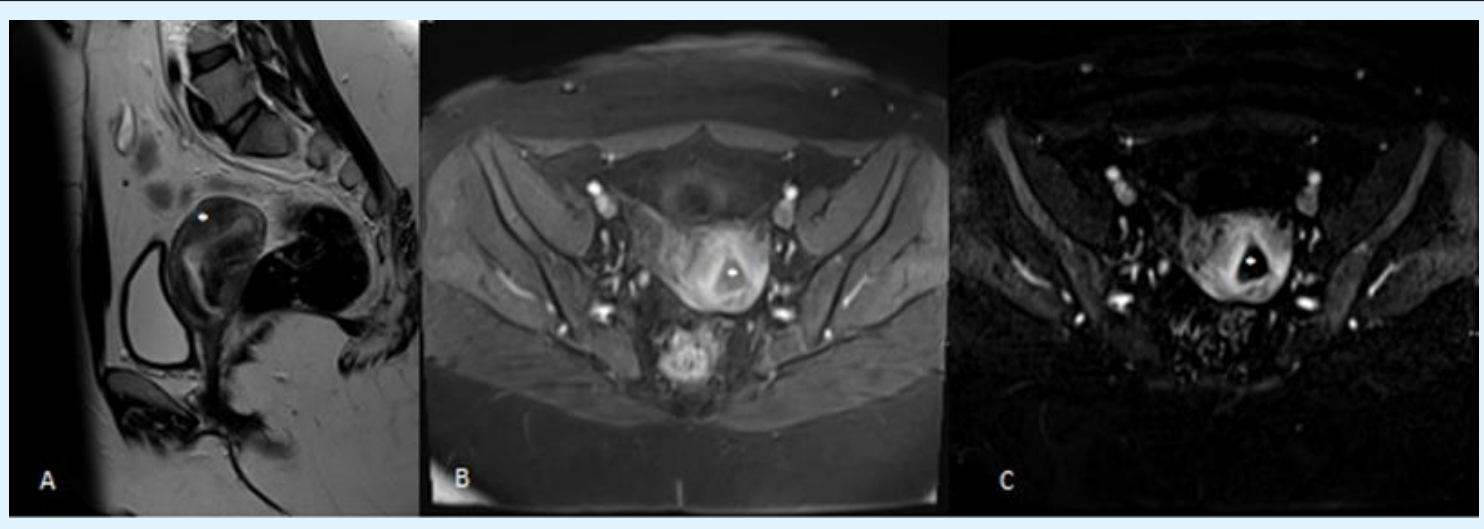

Figure 4: Sagittal (A) T2-wieatghed image, Axial (B) T1-wieatghed image post contrast and (C) subtraction of the pelvis at the level of the uterus post treatment show normalized zonal anatomy of the uterus with disappear of the multiple large serpentine endometrial vessels (white star in A, B and C).

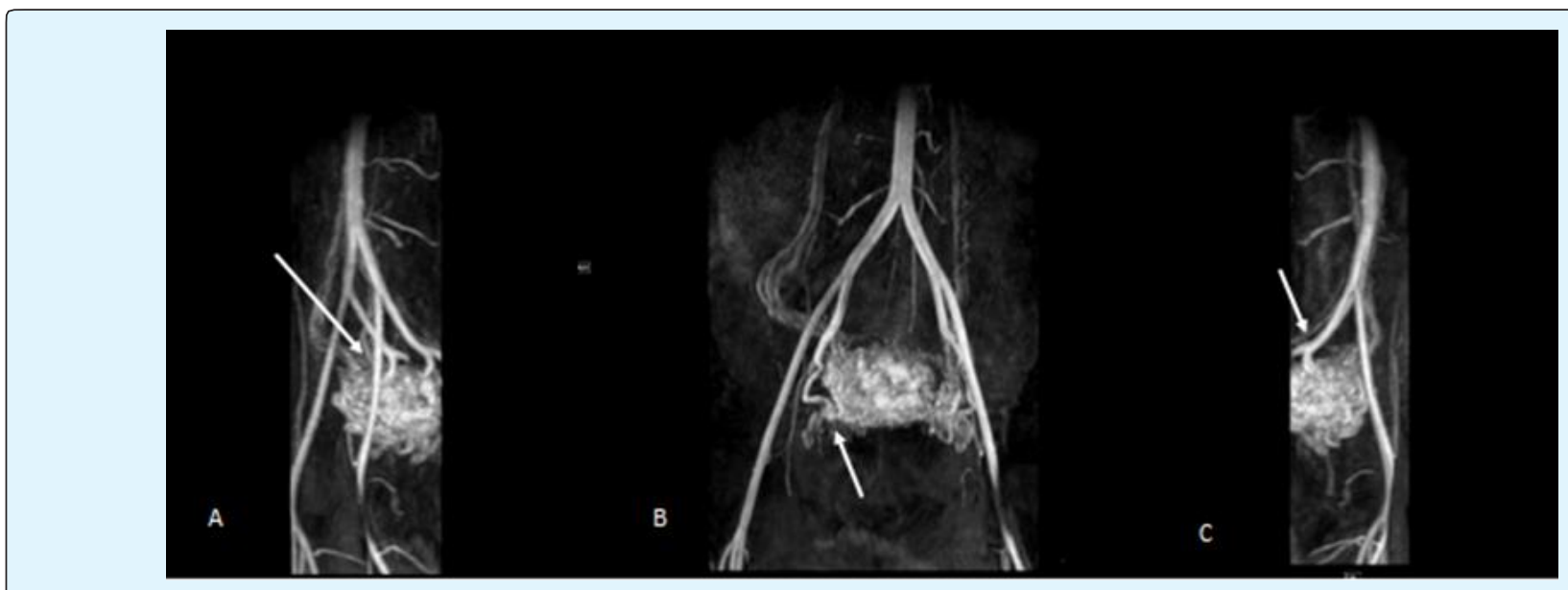

Figure 5: Sagittal A, B and coronal C Contrast-enhanced MR angiography of pelvic show multiple large serpentine abnormal vessels arising from bilateral internal illac vessels (white arrow in A, B and C). 


\section{Medical Journal of Clinical Trials \& Case Studies}

\section{Discussion}

A review of the literature discovers inconsistency in fertility outcome after UAE (uterine artery embolization) for post-partum hemorrhage and symptomatic uterine fibroids. Increased risk of spontaneous abortion and postembolization uterine necrosis had been reported in many studies [17-19]. Repeated embolization rate was $17 \%$ while future pregnancy rate was quoted as $29 \%$ in one study [20].

In 2013, ACOG and the Society of Interventional Radiology warned that uterine artery embolization should be used with caution among those who desire future childbearing, as the impact on fertility is still unclear [8]. Most Patients with AVM are in the reproductive age group. Possible Impact on future child bearing with the use of UAE has opened a debate to seek safer options that can be used electively to treat AVM.

There are cases where UAE proved extremely effective in treating AVM especially in Hemodynamically unstable patients, like in our patient it was almost lifesaving. In contrast Calzolari $\mathrm{S}$, et al. performed a retrospective study on 11th cases diagnosed with uterine arteriovenous malformation and treated with surgical hysteroscopy reported high fertility outcomes, a $100 \%$ success rate after the first treatment, no complications related to the surgical procedure and no hospital admission [21]. The surgical procedure time is short and the patient is not exposed to ionizing radiation [21]. However the use of hysteroscopy required hemodynamic ally stable patients.

There are reported successful cases of laparoscopic bipolar coagulation as well. Parson and co did a systemic review of 21 studies describing pregnancy and complications of pregnancy after UAE $[8,15,16]$. Low level evidence from these studies suggests that pregnancy rate following UAE is comparable to the age adjusted rates in general population. Although pregnancy complication like miscarriage was higher with UAE.

The TOG published an article on pregnancy outcome after uterine artery embolization for fibroids [22]. After reviewing the literature author conclude that weight of retrospective data and small prospective trials of UAE v/s surgical intervention suggest increased level of adverse pregnancy outcome following embolization. There was non-significant trend towards higher rate of miscarriage, abnormal presentation, preterm delivery, fetal growth restriction and postpartum Hemorrhage.

As there is no clear consensus on the best treatment for AVM. The current medical and surgical options are based on clinician's experience and published case reports. The treatment depends on the extent of bleeding. In mild to moderate cases with hemodynamic ally stable patient, the first clinical approach would be conservative for a maximum period of 3-6 months [8]. Persistent bleeding, failed conservative management and unstable patient warrant embolization of the uterine or iliac artery which effectively treat the lesion $[10,11]$.

Women seeking fertility should be informed about the uncertain and inconsistent effect of UAE on fertility. Less frequently used surgical methods, such as the coagulation of AVM under hysteroscopic guidance, laparoscopic bipolar coagulation of uterine vessels and surgical removal of the AVM could be considered an option for women who retain reproductive ambitions $[23,24]$.

\section{Conclusion}

Although uterine artery embolization is the cornerstone of the treatment of AVM in case of bleeding and hemodynamic instability, further randomized controlled trial comparing UAE versus other fertilitysparing treatments are necessary to confirm the usefulness of these modalities in patients with a desire for future child bearing.

\section{References}

1. Diwan RV, Brennan JN, Selim MA, McGrew TL, Rashad FA, et al. (1983) Sonographic diagnosis of arteriovenous malformation of the uterus and pelvis. J Clin Ultrasound 11(5): 295-298.

2. Fleming H, Ostor A, Pickel H, Fortune D (1989) Arteriovenous malformations of the uterus. Obstet Gynaecol 73: 209-213.

3. Huang MW, Muradali D, Thurston WA, Burns PN, Wilson SR (1998) Uterine arteriovenous malformations: gray-scale and Doppler US features with MR imaging correlation. Radiology 206(1): 115123. 


\section{Medical Journal of Clinical Trials \& Case Studies}

4. Kasznica J, Nisar N (1995) Congenital vascular malformation of the uterus in a stillborn: a case report. Hum Pathol 26(2): 240-241.

5. Vijayakumar A, Srinivas A, Chandrashekar BM, Vijayakumar A (2013) Uterine vascular lesions. Rev Obstet Gynecol 6(2): 69-79.

6. Timmerman D, Wauters J, Van Calenbergh S, Van Schoubroeck D, Maleux G, et al. (2003) Color Doppler imaging is a valuable tool for the diagnosis and management of uterine vascular malformations. Ultrasound Obstet Gynecol 21(6): 570-577.

7. Gopal M, Goldberg J, Klein TA, Fossum GT (2003) Embolization of a uterine arteriovenous malformation followed by a twin pregnancy. Obstet Gynecol 102(4): 696-698.

8. Mohan PP, Hamblin MH, Vogelzang RL (2013) Uterine artery embolization and its effect on fertility. J Vasc Interv Radiol 24(7): 925-930.

9. Nonaka T, Yahata T, Kashima K, Tanaka K (2011) Resolution of uterine arteriovenous malformation and successful pregnancy after treatment with a gonadotropin-releasing hormone agonist. Obstet Gynecol 117(2): 452-455.

10. Patton EW, Moy I, Milad MP, Vogezang R (2015) Fertility-preserving management of a uterine arteriovenous malformation: a case report of uterine artery embolization (UAE) followed by laparoscopic resection. J Minim Invasive Gynecol 22(1): 137-141.

11. O'Brien P, Neyastani A, Buckley AR, Chang SD, Legiehn GM (2006) Uterine arteriovenous malofrmations: from diagnosis to treatment. J Ultrasound Med 25(11): 1387-1392.

12. Yang JJ, Xiang Y, Wan XR, Yang XY (2005) Diagnosis and management of uterine arteriovenous fistulas with massive vaginal bleeding. Int J Gynaecol Obstet 89(2): 114-119.

13. Sanguin S, Lanta-Delmas S, Le Branche A, GrardelChambenoit E, Merviel P, et al. (2011) Uterine arteriovenous malformations: diagnosis and treatment in 2011. Gynecol Obstet Fertil 39(12): 722 727.
14. Wu YC, Liu WM, Yuan CC, Ng HT (2001) Successful treatment of symptomatic arteriovenous malformation of the uterus using laparoscopic bipolar coagulation of uterine vessels. Fertil Steril 76(6): 1270-1271.

15. Manolitsas T, Hurley V, Gilford E (1994) Uterine arteriovenous malformation- -a rare cause of uterine haemorrhage. Aust N Z J Obstet Gynaecol 34(2): 197199.

16. Milingos D, Doumplis D, Sieunarine K, Savage $P$, Lawson AD, et al. (2007) Uterine arteriovenous malᄀformation: fertility-sparing surgery using unilateral ligation of uterine artery and ovarian ligament. Int J Gynecol Cancer 17(3): 735-737.

17. Holub Z, Mara M, Kuzel D, Jabor A, Maskova J, et al. (2008) Pregnancy outcomes after uterine artery occlusion: prospective multicentric study. Fertil Steril 90(5): 1886-1891.

18. Homer H, Saridogan E (2010) Uterine artery embolization for fibroids is associated with an increased risk of miscarriage. Fertil Steril 94(1): 324330 .

19. Poujade O, Ceccaldi PF, Davitian C, Amate P, Chatel P, et al. (2013) Uterine necrosis following pelvic arterial embolization for post-partum hemorrhage: review of the literature. Eur J Obstet Gynecol Reprod Biol 170(2): 309-314.

20. Peitsidis P, Manolakos E, Tsekoura V, Kreienberg R, Schwentner L (2011) Uterine arteriovenous malformations induced after diagnostic curettage: a systematic review. Arch Gynecol Obstet 284(5): 1137-1151.

21. Calzolari S, Cozzolino M, Castellacci E, Dubini V, Farruggia A, et al. (2017) Hysteroscopic Management of Uterine Arteriovenous Malformation. JSLS 21(2).

22. Homer H, Saridogan E (2009) Review pregnancy outcome after uterine artery embolization for fibroid. The obstetrician and gynecologist 11: 265-270.

23. Ahmed Samy El Agwany, Mohamed Elshafei (2018) Extensive uterine arteriovenous malformation with hemodynamic instability- Embolization for whole myometrium affection. EJOG 222: 188-191. 


\section{Medical Journal of Clinical Trials \& Case Studies}

24. Vedantham S, Goodwin SC, McLucas B, Mohr G (1997) Uterine artery embolization: an underused method of controlling pelvic hemorrhage. Am J Obstet Gynecol 176(4): 938-948. 